\title{
Multitemperature blackbody spectra of thin accretion disks with and without a zero-torque inner boundary condition
}

\section{Citation}

Zimmerman, E. R., R. Narayan, J. E. McClintock, and J. M. Miller. 2005. “Multitemperature Blackbody Spectra of Thin Accretion Disks with and without a Zero\#Torque Inner Boundary Condition." The Astrophysical Journal 618 (2): 832-44. https://doi.org/10.1086/426071.

\section{Permanent link}

http://nrs.harvard.edu/urn-3:HUL.InstRepos:41384968

\section{Terms of Use}

This article was downloaded from Harvard University's DASH repository, and is made available under the terms and conditions applicable to Other Posted Material, as set forth at http:// nrs.harvard.edu/urn-3:HUL.InstRepos:dash.current.terms-of-use\#LAA

\section{Share Your Story}

The Harvard community has made this article openly available.

Please share how this access benefits you. Submit a story.

Accessibility 


\title{
Multi-Temperature Blackbody Spectra of Thin Accretion Disks With and Without a Zero-Torque Inner Boundary Condition
}

\author{
E. R. Zimmerman, R. Narayan, J. E. McClintock, and J. M. Miller \\ Harvard-Smithsonian Center for Astrophysics, 60 Garden Street, Cambridge, MA 02138
}

\begin{abstract}
The standard spectral model for analyzing the soft component of thermal emission from a thin accretion disk around a black hole is the multi-temperature blackbody (MTB) model. The widely used implementation of this model, which is known as "diskbb," assumes nonzero torque at the inner edge of the accretion disk. This assumption is contrary to the classic and current literature on thindisk accretion, which advocates the use of a zero-torque boundary condition. Consequently, we have written code for a zero-torque model, "ezdiskbb," which we compare to the nonzero-torque model diskbb by fitting $R X T E$ spectra of three well-known black hole binaries: 4U 1543-47, XTE J1550-564, and GRO J1655-40. The chief difference we find is that the zero-torque model gives a value for the inner disk radius that is $\approx 2.2$ times smaller than the value given by diskbb. This result has important implications, especially for the determination of black-hole angular momentum and mass accretion rate.
\end{abstract}

Subject headings: accretion, accretion disks - black hole physics - X-ray: stars — stars: individual (4U 1543-47, XTE J1550-564, GRO J1655-40)

\section{Introduction}

One of the most widely used models in studies of the spectra of accretion disks in black hole X-ray binaries is the multi-temperature blackbody (MTB) model, which has been standard in the literature for over 30 years (Pringle \& Rees 1972; Shakura \& Sunyaev 1973; Pringle 1981; Frank, King \& Raine 1992). In many systems, an $L_{X} \propto T^{4}$ dependence has been observed (see, e.g., Gierliński \& Done 2004), confirming a fundamental prediction of such models and demonstrating that disks are an important and prevalent accretion structure in black hole systems. Fitting spectra with the MTB model allows us to determine important properties of accretion disks, including accretion rate, inner radius, and temperature. This information about the accretion disk can in principle be used to deduce the 
angular momentum of the black hole (e.g., Zhang, Cui, \& Chen 1997), and the radius at which an accretion disk might be truncated in low accretion rate phases (e.g., Esin et al. 2001).

Over the years, a number of different assumptions have been used in deriving the MTB spectrum. A standard assumption in the literature is that a zero-torque boundary condition should be applied to the inner edge of the accretion disk (Shakura \& Sunyaev 1973; Novikov \& Thorne 1973). Gierliński et al. (1999) have shown, however, that the widely used MTB model known as "diskbb" (see, e.g., Mitsuda et al. 1984) that is found in various X-ray spectral fitting packages assumes a nonzero torque at the inner boundary of the disk.

In order to test the effects of zero-torque and nonzero-torque inner disk boundary conditions, we have constructed disk models for each scenario and fit a number of black hole binary spectra. In section 2, we outline the MTB model and illustrate the difference in the temperature profiles that are obtained when the zero-torque inner boundary condition is and is not used. In section 3, we analyze the spectra of three well-known black hole X-ray novae (BHXN): 4U1543-47, XTE J1550-564, and GRO J1655-40. Section 4 discusses the implications of the results, and section 5 presents a summary and some conclusions.

\section{Multi-Temperature Disk Blackbody Model}

In this paper, we are interested in the multi-temperature blackbody spectrum of a thin accretion disk around a compact star. Following standard treatments of this problem (e.g., Pringle 1981, Frank et al. 1992) we assume Newtonian gravity and take the disk to be in steady state. We limit ourselves to a brief discussion of the main results, referring the reader to the literature cited above for details.

A thin accretion disk has a negligible radial pressure gradient. Therefore, at each radius $R$, the gas orbits at essentially the Keplerian angular frequency, $\Omega_{K}=\left(G M / R^{3}\right)^{1 / 2}$, where $M$ is the mass of the central star. Internal viscous torques in the accreting gas transfer angular momentum outward, allowing the material to spiral in. These torques dissipate energy, and the dissipation rate per unit surface area is given by

$$
D(R)=\frac{1}{2} \nu \Sigma\left(R \Omega_{K}^{\prime}\right)^{2}
$$

where $\nu$ is the kinematic coefficient of viscosity, $\Sigma$ is the surface density, $\Omega_{K}^{\prime}=d \Omega_{K} / d R=$ $-3 \Omega_{K} / 2 R$, and the factor of $1 / 2$ takes into account the two disk surfaces, one above and one below the mid-plane. The mass accretion rate is given by $\dot{M}=2 \pi R \Sigma\left(-v_{R}\right)=$ constant,

where $v_{R}$ is the radial drift velocity of the gas (negative for inward flow). Conservation of 
angular momentum is then expressed by the condition

$$
-\nu \Sigma \Omega_{K}^{\prime}=-v_{R} \Sigma \Omega_{K}+\frac{C}{2 \pi R^{3}},
$$

where $C$ is a constant of integration. To determine the value of $C$, we apply a boundary condition on the disk; specifically, we assume a value for the torque at the inner edge of the disk, located at $R=R_{\text {in }}$. The standard choice is a zero-torque boundary condition (e.g., Pringle 1981; Frank et al. 1992), which is appropriate in the following two situations.

First, if the accretion disk is terminated on the inside by a star spinning at a rate below the break-up limit, then there must be a radius close to the surface of the star at which the angular velocity of the orbiting gas reaches a maximum, meaning that $\Omega_{K}^{\prime}=0$. At this radius, since the shear is zero, the viscous shear stress must vanish. Thus, if we identify this radius as the inner edge of the disk $R_{\text {in }}$, then the torque at the inner edge clearly vanishes. In this picture, the accretion disk is identified as the region $R \geq R_{\text {in }}$ and it has a zerotorque inner boundary condition, while the region between $R_{\text {in }}$ and the surface of the star is called the boundary layer. In what follows, we ignore the boundary layer, whose radiation is typically much harder than the disk emission.

The second situation in which the zero-torque boundary condition is valid is when a thin accretion disk extends down to the marginally stable orbit at radius $R_{\mathrm{ms}}$ around a black hole or very compact neutron star. In such a disk, gas spirals in slowly and viscously down to $R=R_{\mathrm{ms}}$ and then free-falls rapidly with negligible viscous interactions. If the accretion disk is thin, the transition from viscous to dynamical flow occurs quite suddenly (Afshordi \& Paczyński 2003). We may then identify $R_{\mathrm{in}}=R_{\mathrm{ms}}$ as the inner edge of the disk, and we may safely assume a nzero-torque boundary condition at this radius.

Since the torque at $R=R_{\text {in }}$ vanishes, the right-hand side of equation (2) is zero. This determines the value of $C$, and it is then straightforward to solve for $D(R)$. Let us assume that the disk is optically thick and that it radiates as a modified blackbody with a radial temperature profile $T(R)$. Then we have

$$
\sigma\left[\frac{T(R)}{f}\right]^{4}=D(R)=\frac{3 G M \dot{M}}{8 \pi R^{3}}\left[1-\left(\frac{R_{\text {in }}}{R}\right)^{1 / 2}\right],
$$

where $\sigma$ is the Stefan-Boltzmann constant, and the spectral hardening factor $f$ accounts approximately for the modification of the optically thick disk emission from a pure blackbody. The modification could occur because of the combined effect of scattering and absorptive opacity (e.g., Zavlin, Pavlov \& Shibanov 1996; Rutledge et al. 1999; McClintock, Narayan \& Rybicki 2004), or due to Comptonization (Shimura \& Takahara 1995). Neither effect is fully described by a simple rescaling of the temperature of the spectrum by a single factor 
$f$, nor is $f$ expected to be independent of radius. For simplicity, however, we make both assumptions in writing equation (3). Note that for a canonical blackbody spectrum, $f=1$.

We refer to the temperature defined by the equation above as $T_{\mathrm{zt}}$ to indicate that it is derived with the zero-torque boundary condition. Defining a dimensionless radius, $r=R / R_{\text {in }}$, we thus have

$$
T_{\mathrm{zt}}(r)=T_{\star} r^{-3 / 4}\left(1-r^{-1 / 2}\right)^{1 / 4}, \quad T_{\star}=f\left(\frac{3 G M \dot{M}}{8 \pi R_{i n}^{3} \sigma}\right)^{1 / 4} .
$$

The parameter $T_{\star}$ is, however, not a very convenient quantity since the disk does not achieve this temperature at any radius. Let us, therefore, re-express equation (4) in terms of the maximum temperature of the disk, $T_{\max }$ :

$$
T_{\mathrm{zt}}(r)=2.05 T_{\max } r^{-3 / 4}\left(1-r^{-1 / 2}\right)^{1 / 4}, \quad T_{\max }=0.488 T_{\star}, \quad r \equiv \frac{R}{R_{\mathrm{in}}} .
$$

Finally, by integrating the Planck function (with spectral hardening factor $f$ ) over the area of the disk, and assuming a distance $D$ and inclination $i$ for the source, we obtain the observed spectral flux in units of erg $\mathrm{s}^{-1} \mathrm{~cm}^{-2} \mathrm{keV}^{-1}$ :

$$
F_{E}=K \frac{4 \pi E^{3}}{h^{3} c^{2}} \int_{1}^{\infty} \frac{r}{e^{E / k T_{\mathrm{zt}}(r)}-1} d r
$$

where

$$
K=\frac{1}{f^{4}}\left(\frac{R_{\text {in }}}{D}\right)^{2} \cos i .
$$

From equations (5)-(7), we see that the spectrum is determined by one parameter, $T_{\max }$, and that it has a normalization constant, $K$, which depends on four system-specific quantities: the inner radius of the disk, the inclination of the disk, the distance to the source, and the spectral hardening factor.

By integrating $D(R)$ over the entire disk (top and bottom surfaces) from $R=R_{\text {in }}$ to $R_{\text {out }} \rightarrow \infty$, we may calculate the total luminosity of the disk:

$$
L_{\text {disk }}=\frac{G M \dot{M}}{2 R_{\text {in }}}=73.9 \sigma\left(\frac{T_{\max }}{f}\right)^{4} R_{\text {in }}^{2} \quad \text { (zero torque). }
$$

Note that only half the gravitational binding energy of the accreting gas is radiated by the disk; the other half survives as kinetic energy of the gas at the inner edge of the disk. In the case of accretion onto a star, some or all of this residual energy is dissipated in the boundary layer as the gas spins down to merge with the surface of the star (see Kluzniak 1987; Popham 
\& Narayan 1995 for an estimate of the fraction of this energy that is radiated for a given stellar spin). In the case of accretion onto a black hole, the energy remains in the gas and falls into the hole.

The above discussion is for a disk with a zero-torque inner boundary condition. In some cases, however, a disk may have a finite torque at its inner edge. For instance, Popham \& Narayan (1991) and Paczyński (1991) showed that a star that has been spun up to breakup can lose angular momentum to a surrounding accretion disk via a torque at the interface. The torque can (in principle) have any magnitude. In the case of a disk around a black hole, some authors have recently discussed the possibility that magnetic fields may induce a nonzero torque at the marginally stable orbit (Gammie 1999; Agol \& Krolik 2000; Reynolds \& Armitage 2001). Afshordi \& Paczyński (2003) argue that the magnitude of the torque at $R_{\mathrm{ms}}$ depends on the vertical thickness of the disk: whereas a thick disk could have a substantial torque, a thin disk should at most have only a very weak torque (see also Armitage, Reynolds \& Chiang 2001).

The study of disks with nonzero torque is hampered by the fact that there is no characteristic or "natural" magnitude that one can select for the torque. A complete analysis will thus need to include the strength of the torque as a second parameter. In this paper, we consider a particular choice for the torque, corresponding to the case when the integration constant $C$ in equation (2) is zero. This is to be viewed as just an example, though as we discuss in $\S 3.1$, this particular case is historically important. When $C=0$, the temperature profile takes the simple form

$$
T_{\mathrm{st}}(r)=T_{\star} r^{-3 / 4}=T_{\max } r^{-3 / 4}
$$

where $T_{\star}$ is again defined as in equation (4), and the subscript in $T_{\text {st }}$ stands for "standard torque." The profiles $T_{\mathrm{zt}}(r)$ and $T_{\mathrm{st}}(r)$ are compared in Figure 1 . We see that $T_{\mathrm{zt}}(r)$ goes to zero at the inner edge of the disk since the torque vanishes there, whereas $T_{\mathrm{st}}(r)$ reaches its maximum value at the inner edge because the torque is maximum.

The spectrum of a disk with the standard-torque temperature profile is given by equation (6), except that $T_{\mathrm{zt}}(r)$ is replaced by $T_{\mathrm{st}}(r)$. The total disk luminosity is

$$
L_{\text {disk }}=\frac{3 G M \dot{M}}{2 R_{\text {in }}}=12.6 \sigma\left(\frac{T_{\max }}{f}\right)^{4} R_{\text {in }}^{2} \quad \text { (standard torque). }
$$

Note that for given $M, \dot{M}$, and $R_{\text {in }}$, this disk model has approximately twice the maximum temperature and exactly three times the luminosity of a disk with zero torque. The extra luminosity is because of the work done by the nonzero torque at the inner edge. Figure 2 shows the MTB spectra of the two temperature models presented in Figure 1 . There is a 
significant difference between the two spectra, especially for photon energies greater than 1 $\mathrm{keV}$ (for this particular example), where the $T_{\mathrm{st}}$ model has substantially more emission.

\section{Analysis and Results}

\section{1. $\quad$ Disk Models}

The X-ray spectral fitting package XSPEC (Arnaud 1996) contains diskbb, a widely used version of the MTB spectral model. As we stated in the introduction, Gierliński et al. (1999) have pointed out that diskbb assumes a nonzero torque at the inner boundary of the accretion disk. The help manual on diskbb within XSPEC and the papers to which it refers (Mitsuda et al. 1984; Makishima et al. 1986) are ambiguous as to whether Gierliński et al.'s assertion is true. The latter papers refer to Pringle (1981), which describes both the zerotorque temperature profile (eq. 4 in our paper), and the standard-torque profile (eq. 9). In private correspondence, Makishima, one of the originators of the diskbb code, confirmed that the standard-torque condition is assumed by diskbb. We decided to check this for ourselves. We wrote a code called diskbbcheck, which explicitly uses $T_{\mathrm{st}}(r)$ as the temperature profile and, as described below, we compared the results obtained using diskbbcheck against those obtained with diskbb.

We also wrote code for another model, which we call ezdiskbb. This model corresponds to the zero-torque boundary condition at the inner edge of the disk, and makes use of the temperature profile $T_{\mathrm{zt}}(r)$. We used this code to compare the results of spectral fits done with and without the zero-torque inner boundary condition, as discussed below.

Each of the models, diskbb, diskbbcheck, and ezdiskbb has two adjustable parameters. The first is the maximum temperature in the disk, which we call $T_{\max }$. When the standard-torque boundary condition is assumed, $T_{\max }$ is identical to $T_{\star}$, the temperature at the inner edge of the accretion disk (Fig. 1). The second parameter in all three models is a normalization constant, $K$, which is defined as follows:

$$
K=\frac{1}{f^{4}}\left(\frac{R_{\text {in }} / 1 \mathrm{~km}}{D / 10 \mathrm{kpc}}\right)^{2} \cos i .
$$

The current version of diskbb implicitly assumes a spectral hardening factor of $f=1$ in its normalization. A summary of the three models is given in Table 1. 


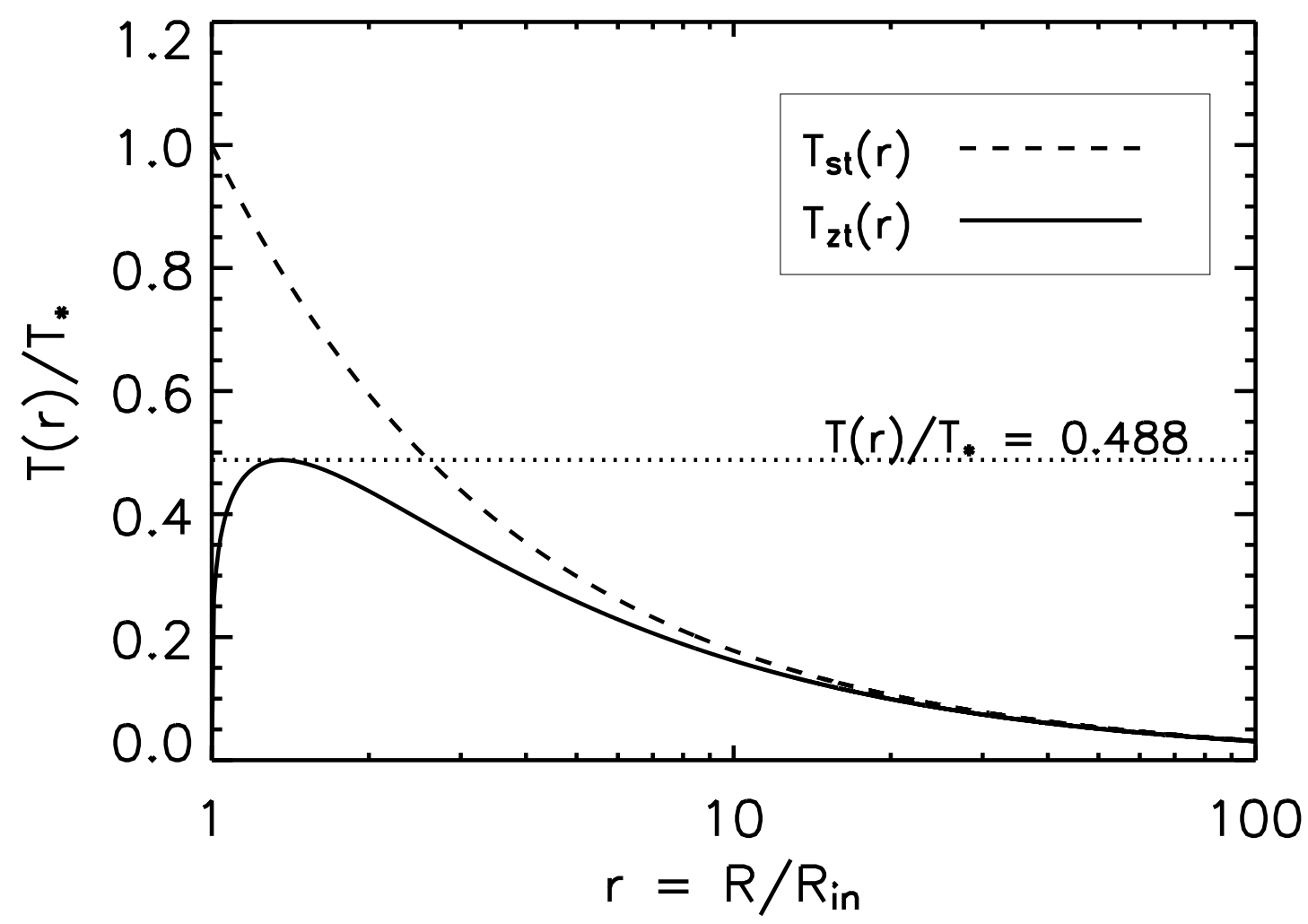

Fig. 1.- Disk temperature as a function of radius for the two models discussed in $\S 2$. The vertical axis is in units of $T / T_{\star}$, while the horizontal axis is in units of $r=R / R_{\mathrm{in}}$. The solid curve shows the profile $T_{\mathrm{zt}}(r)$ of a disk with a zero-torque boundary condition at the inner edge of the disk (eq. 4). The dashed curve shows the profile $T_{\mathrm{st}}(r)$ of the standard-torque model (eq. 9).

Table 1. MTB Models

\begin{tabular}{cccc}
\hline \hline Model & BC & Description & References \\
\hline diskbb & Standard-torque & Standard XSPEC model & Mitsuda et al. (1984); Makishima et al. (1986) \\
diskbbcheck & Standard-torque & Confirmed BC in diskbb & This work \\
ezdiskbb & Zero-torque & Alternative to diskbb & This work \\
\hline
\end{tabular}




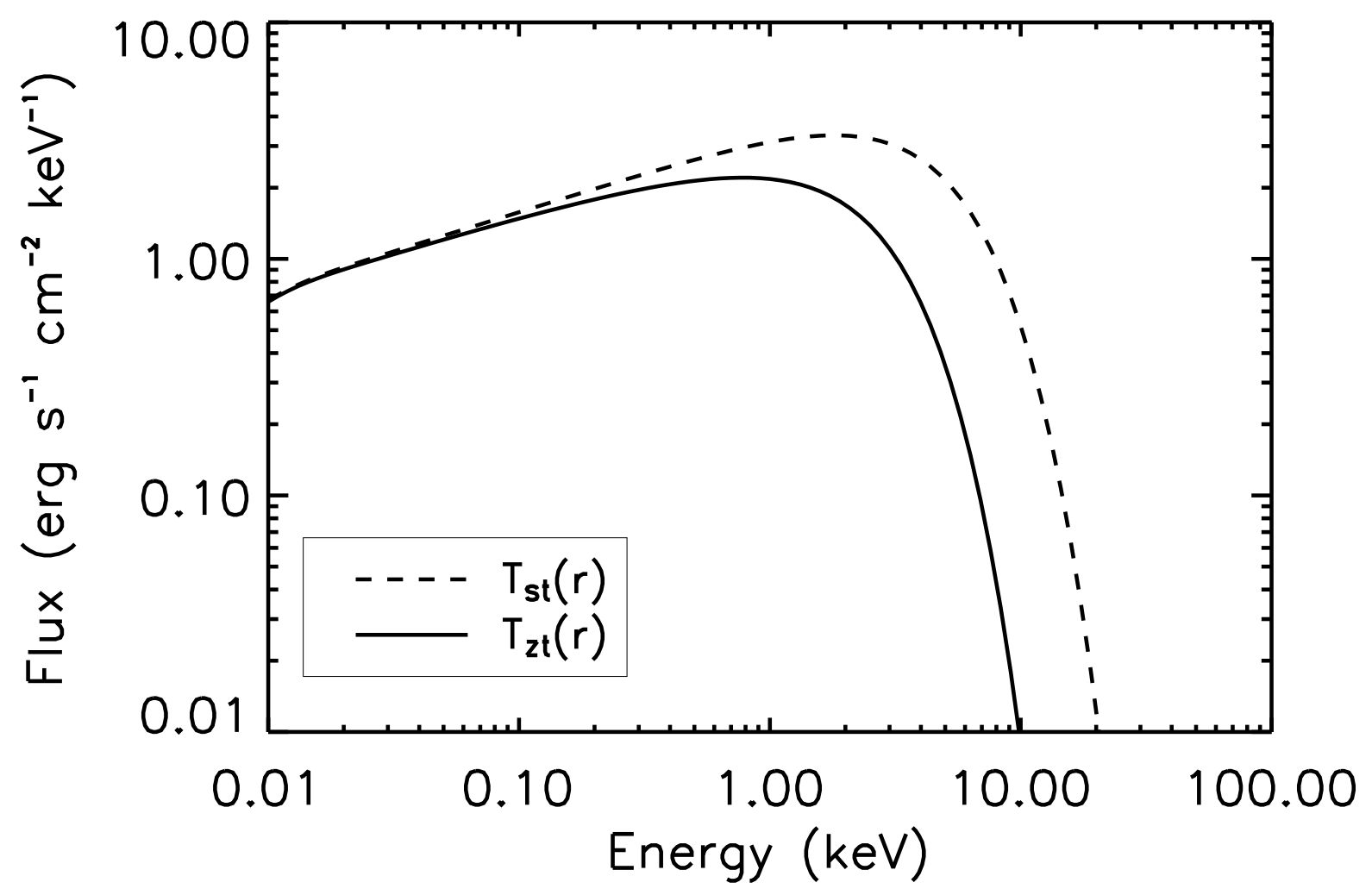

Fig. 2.- MTB disk spectra corresponding to the two temperature profiles shown in Figure 1. The following parameters have been assumed: $T_{\star}=2 \mathrm{keV}, R_{\mathrm{in}}=10 \mathrm{~km}, D=10 \mathrm{kpc}, i=$ 0 degrees, $f=1$. Note the large difference between the two spectra, especially for photon energies above $1 \mathrm{keV}$. 


\subsection{Analysis}

We compared the performance of the two models by analyzing data for three BHXN that were obtained using the Proportional Counter Array (PCA) aboard the Rossi X-ray Timing Explorer (RXTE). The PCA consists of five xenon-filled proportional counter units (PCUs) that have a total effective area of $6200 \mathrm{~cm}^{2}$ at $5 \mathrm{keV}$ (Jahoda et al. 1996). The PCA is sensitive over $2-60 \mathrm{keV}$, with an effective energy range of $\sim 2.5-20 \mathrm{keV}$ and an energy resolution of $\sim 17 \%$ at $5 \mathrm{keV}$ (Sobczak 2000). We also used lightcurves obtained using RXTE's All-Sky Monitor (ASM), which scans $\sim 80 \%$ of the sky during every orbit (Sobczak 2000). The ASM has three energy channels: $1.5-3 \mathrm{keV}, 3-5 \mathrm{keV}$, and $5-12 \mathrm{keV}$ and is very useful for identifying and monitoring transient sources like BHXN.

In our analysis, we used PCA data for three BHXN: 4U1543-47 (hereafter U1543), XTE J1550-564 (hereafter J1550), and GRO J1655-40 (hereafter J1655). The BH mass, distance, and inclination have been determined through optical observations for each of these objects. These properties are given in Table 2 .

These three objects have all had recent outbursts that have been analyzed in detail using RXTE observations. Park et al. (2003) studied the 2002 outburst of U1543; Sobczak et al. (2000) analyzed the outburst of J1550 in 1998-1999; and Sobczak et al. (1999) analyzed J1655's 1996-1997 outburst. The ASM lightcurves of each of these outbursts, along with their hardness ratios (HR2) versus time, are shown in Figure 3. The values for HR2 correspond to the $5-12 \mathrm{keV}$ intensity divided by the $3-5 \mathrm{keV}$ intensity, as measured by the ASM. For each of these sources, we chose 10 observations to analyze. All of these observations were chosen when the source was in what is canonically known as its "high/soft" state, during which the soft thermal component from a hot disk dominates the spectrum (McClintock \& Remillard 2004). This state corresponds approximately to a hardness ratio of 0.5 . The times of our chosen observations are denoted by the dashed vertical lines in Figure 3.

In fitting the spectra of these sources, we followed as closely as possible the procedures described in the papers cited above. In addition to the MTB models, we used four other XSPEC models in our fits. Primary among these was the power-law model, which has two parameters: the photon index, $\Gamma$, and a normalization constant, which we will call $K_{\mathrm{PL}}$. The next component that we used was an interstellar absorption model, which has one parameter: the hydrogen column density, $N_{\mathrm{H}}$, in units of atoms $\mathrm{cm}^{-2}$. The third component in our fits was a smeared absorption edge model ("smedge"), which has three parameters: the threshold energy in $\mathrm{keV}$, the smearing width in $\mathrm{keV}$, and the maximum absorption factor at threshold, $\tau_{\mathrm{FE}}$ (the smedge model does have one additional parameter, the index for photoelectric crosssection, but this parameter always remains fixed at a value of -2.67). Our final spectral component was a Gaussian, which was used to fit an Fe line feature for U1543 and J1550, 

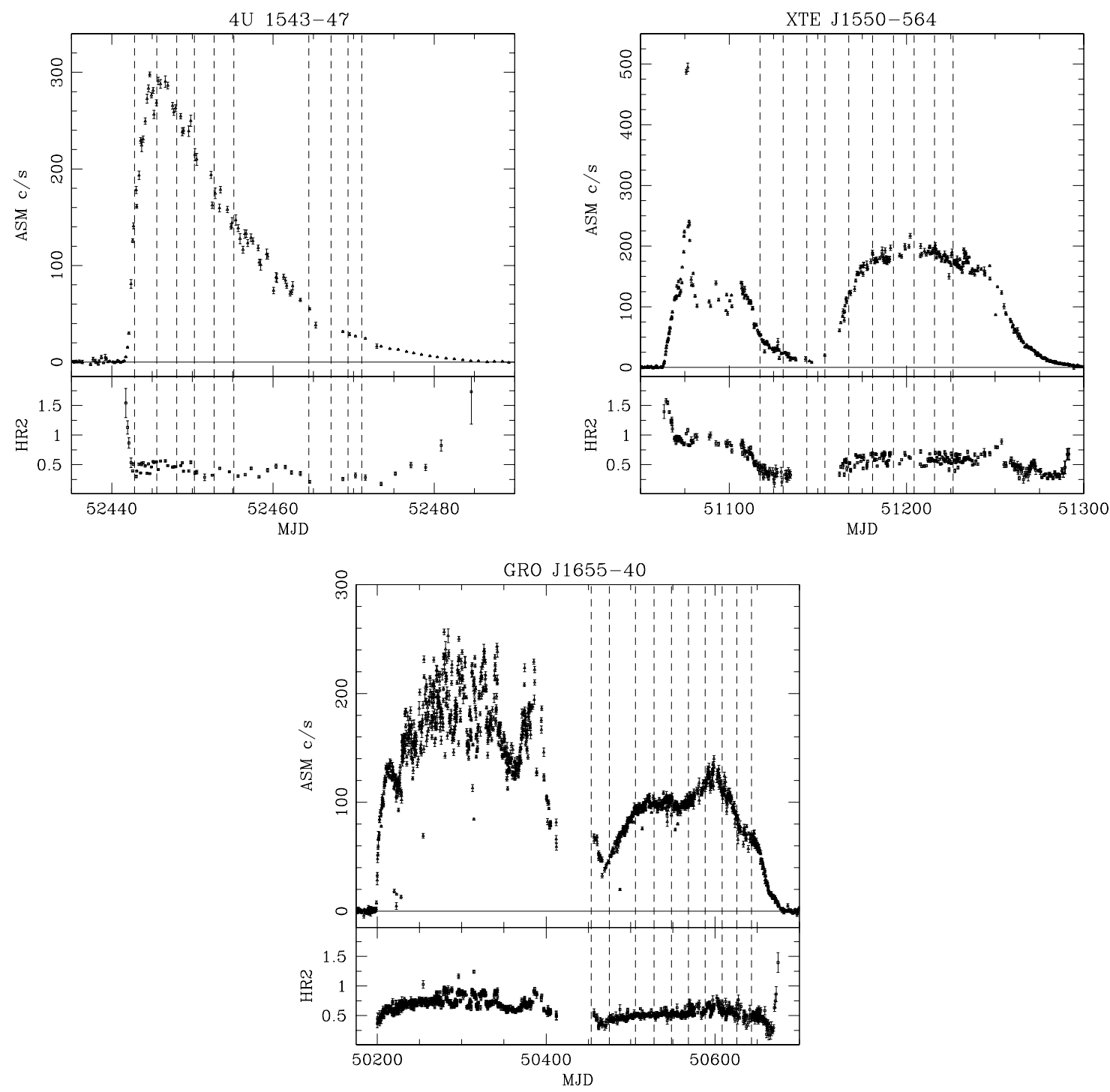

Fig. 3.- The 1.5-12 keV intensities and hardness ratios of 4U 1543-47, XTE J1550-564, and GRO J1655-40 plotted versus time, as observed by the RXTE All-Sky Monitor. An X-ray intensity of 1 Crab corresponds to $75.5 \mathrm{ASM}$ c/s. The hardness ratio is the ratio of the 5-12 $\mathrm{keV}$ intensity to the $3-5 \mathrm{keV}$ intensity. The time axis is in units of MJD = JD - 2,400,000.5. The dashed lines in each plot correspond to the 10 observation times that were analyzed in this paper for each source. Note that all the selected observations occurred when the sources were in their high/soft states, characterized by a hardness ratio of approximately 0.5 . 
but not J1655. The three parameters in this model are the line energy in $\mathrm{keV}$, the line width in $\mathrm{keV}$, and the normalization, which we will call $K_{\text {line }}$.

Because our focus was on the MTB models, we froze a number of the extraneous parameters in these other spectral components to minimize their influence on our fits. We fixed the parameters at average values obtained from the previously cited papers. Whenever a parameter was frozen, it was frozen at the same value for all 10 spectra for each source. Table 3 shows which parameters were allowed to float and which were frozen for each object, along with the values at which they were frozen. A blank field in the table indicates that the given spectral component was not used in fits for that source (i.e., the Gaussian for J1655). Note that the disk models and the power-law model are not included in the table, because the parameters from both of these models were always allowed to float.

We analyzed PCA data from all available PCUs; this was generally 3 of the PCUs for each source. Prior to doing our fits, we added $1 \%$ systematic errors to all of the energy channels of our spectra. We obtained the response matrices for our fits from the HEASARC archives. Table 3 also gives the energy range over which the spectra were fit for each source. Again, the guidelines in the cited papers were followed. For J1550 and J1655, Sobczak et al. $(1999,2000)$ adopted an energy range of $2.5-20 \mathrm{keV}$ for their fits, which was appropriate for the earlier PCA response matrices that they used. However, we were unable to obtain acceptable fits over this energy range using the current response matrices. We therefore raised the lower bound on the energy range to 3.0 and $2.8 \mathrm{keV}$ for J1550 and J1655, respectively.

\subsection{Results}

We first determined the boundary condition used in the standard XSPEC model, diskbb, by fitting our 10 spectra of U1543 with both diskbb and diskbbcheck. For all 10 spectra, we obtained identical results with the two models. We therefore confirmed that diskbb is derived using the standard-torque boundary condition.

We next explored the effects of using the zero-torque boundary condition by fitting the spectra of all three sources using ezdiskbb and diskbb and comparing the fit parameters. We found that ezdiskbb gave values for $T_{\max }$ that were $\approx 5 \%$ lower than those given by diskbb when averaged over all of our fits. Figure 4 illustrates this effect, and Table 4 gives the average ratios between the $T_{\max }$ values obtained using diskbb and ezdiskbb for each of our sources, as well as for the extreme range of the ratios between the two models. In Figure 4, we have plotted the values of $T_{\max }$ for a spectral hardening factor of $f=1.7$, the value that is quoted in Shimura \& Takahara (1995) and that is generally accepted throughout 
Table 2. BHXN Properties

\begin{tabular}{|c|c|c|c|}
\hline & $M_{B H}\left(M_{\odot}\right)$ & $D(\mathrm{kpc})$ & $i\left(^{\circ}\right)$ \\
\hline $\begin{array}{c}73.5_{-2.7}^{+1.9 b} \\
0.08^{c}\end{array}$ & & & \\
\hline $4 \mathrm{U} 1543-47$ & $9.4 \pm 2.0^{\mathrm{a}}$ & $7.5 \pm 1.0^{\mathrm{a}}$ & $20.7 \pm 1.0^{\mathrm{a}}$ \\
\hline XTE J1550-564 & $10.56_{-0.88}^{+1.02 \mathrm{~b}}$ & $5.9_{-3.1}^{+1.7 \mathrm{~b}}$ & $73.5_{-2.7}^{+1.9 \mathrm{~b}}$ \\
\hline GRO 1655-40 & $7.02 \pm 0.22^{\mathrm{c}}$ & $3.2 \pm 0.2^{\mathrm{d}}$ & $69.50 \pm 0.08^{c}$ \\
\hline
\end{tabular}

${ }^{a}$ Orosz et al. (1998); Orosz (2003)

${ }^{\mathrm{b}}$ Orosz et al. (2002)

${ }^{\mathrm{c}}$ Orosz \& Bailyn (1997)

${ }^{\mathrm{d}}$ Hjellming \& Rupen (1995)

Table 3. Spectral Fit Components ${ }^{\mathrm{a}}$

\begin{tabular}{ccccccccc}
\hline \hline & Absorption & \multicolumn{3}{c}{ Smedge } & \multicolumn{3}{c}{ Gaussian } \\
\hline & $\begin{array}{c}N_{H} \\
\left(10^{22} \mathrm{~cm}^{-2}\right)\end{array}$ & $\begin{array}{c}\text { Energy } \\
(\mathrm{keV})\end{array}$ & $\begin{array}{c}\text { Width } \\
(\mathrm{keV})\end{array}$ & $\begin{array}{c}\tau_{F E} \\
\text { Energy } \\
(\mathrm{keV})\end{array}$ & $\begin{array}{c}\text { Width } \\
(\mathrm{keV})\end{array}$ & $K_{\text {line }}$ & $\begin{array}{c}\text { Range of Fits } \\
(\mathrm{keV})\end{array}$ \\
\hline 4U 1543-47 & 0.40 & 7.4 & 7.0 & Float & 6.5 & 1.2 & Float & $2.9-25$ \\
XTE J1550-564 & 2.0 & 8.6 & 7.0 & Float & 6.5 & 1.2 & Float & $3.0-20$ \\
GRO 1655-40 & 0.89 & 8.0 & 7.0 & Float & & & & $2.8-20$ \\
\hline
\end{tabular}

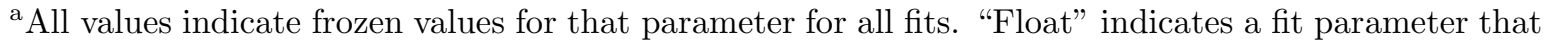
was always allowed to vary. The blank cells for J1655 indicate that a Gaussian was not used in the fits for that object.

${ }^{b}$ Values used for fits were obtained from Park et al. (2003)

${ }^{c}$ Values used for fits were obtained from Sobczak et al. (2000)

${ }^{\mathrm{d}}$ Values used for fits were obtained from Sobczak et al. (1999) 
the literature (see the discussion in $\S 4$ below). The hardening factor relates the observed temperature $T_{\max }$ to the effective temperature: $T_{\text {eff }}=T_{\max } / f$. Note that the hardening factor does not affect the ratio between the diskbb temperature and the ezdiskbb temperature, as long as the same value of $f$ is used in both.

A much more significant effect of using the zero-torque condition was that the values of the inner radius of the disk, $R_{\text {in }}$, were reduced by more than a factor of 2 . Specifically, ezdiskbb gave values for $R_{\text {in }}$ that were a factor of 2.17 smaller on average than those given by diskbb, with the smallest difference between the two models being a factor of 2.15 . This result is shown in Figure 5. The average ratios between the values of $R_{\text {in }}$ for the two models for each source are given in Table 4. The ratios are independent of $f$.

This large difference in the values of $R_{\text {in }}$ between the two models can be explained as follows. In fitting the disk spectrum, the form of which is given in equation (6), XSPEC must fit the shape of the model spectrum - as determined for instance by the peak of the emission - to the observed spectrum. The shape of the MTB model spectrum depends only on the maximum temperature in the disk, and we saw above that diskbb reproduces the observational spectrum with a $T_{\max }$ that is about 1.05 times larger than the corresponding $T_{\max }$ for ezdiskbb. XSPEC must also fit the overall normalization of the model spectrum to the observed one in order to achieve the same luminosity. Equating the luminosities for the two model spectra using eqs. (8) and (10) gives:

$$
\left[73.9 \sigma\left(\frac{T_{\max }}{f}\right)^{4} R_{\mathrm{in}}^{2}\right]_{\mathrm{zt}}=\left[12.6 \sigma\left(\frac{T_{\max }}{f}\right)^{4} R_{\mathrm{in}}^{2}\right]_{\mathrm{st}} .
$$

The right-hand side of equation (12) corresponds to diskbb with its 1.05 times larger value of $T_{\max }$. In order to maintain the equality, this temperature difference requires that $R_{\text {in }}$ be a factor of $\sim 2.2$ larger for diskbb than for ezdiskbb, in agreement with the ratio obtained from the fits (Table 4).

We can also estimate the effect on the estimated mass accretion rate, $\dot{M}$. Comparing equations (8) and (10), we see that for a given $M, \dot{M}$ and $R_{\mathrm{in}}$, the luminosity of the zerotorque model is 3 times less than that of the standard-torque model. Further, we have just shown that $R_{\text {in }}$ for the former model is 2.2 times less compared to the latter model. Therefore, in order to have the same luminosity, the value of $\dot{M}$ with the standard-torque model (diskbb) should be $2.2 / 3 \sim 0.7$ times the value of $\dot{M}$ with the zero-torque model (ezdiskbb). The results of the fits to data are shown in Figure 6, and the average differences in $\dot{M}$ between diskbb and ezdiskbb are given in Table 4 . The results are consistent with the theoretical expectation. 

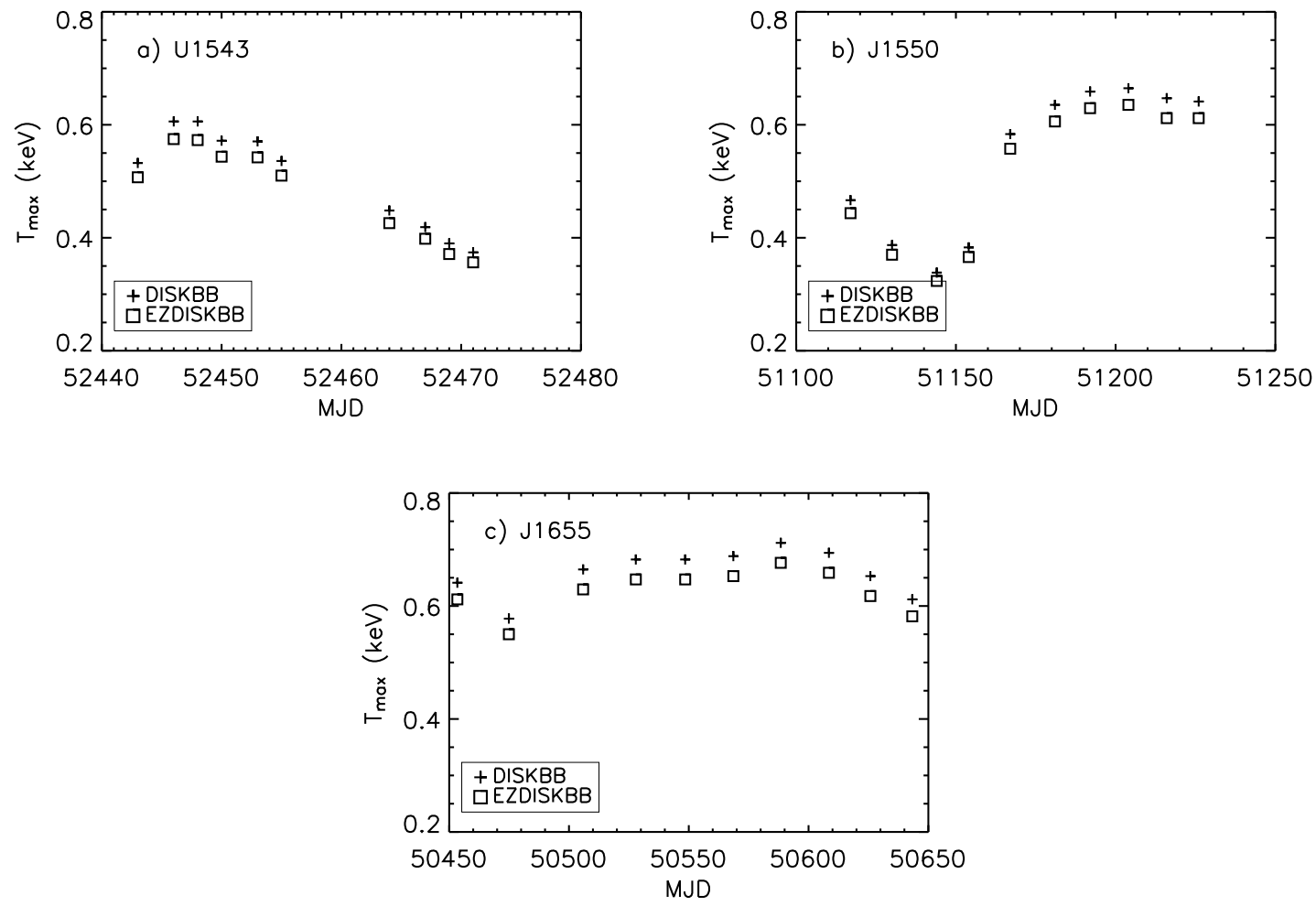

Fig. 4.- $T_{\max }$ versus time for the 10 spectra from each of our BHXN, assuming a spectral hardening factor of $f=1.7$. The horizontal axis gives the date of each observation in units of MJD $=$ JD $-2,400,000.5$. The plus symbols indicate the values of $T_{\max }$ obtained from fits using diskbb, and the squares indicate $T_{\max }$ values from fits using ezdiskbb. We see that ezdiskbb, which assumes a zero-torque boundary condition at the inner edge of the accretion disk, gives values for the maximum temperature in the disk that are always about $5 \%$ lower than those given by diskbb, which assumes the standard-torque inner boundary condition. This ratio is independent of $f$. 

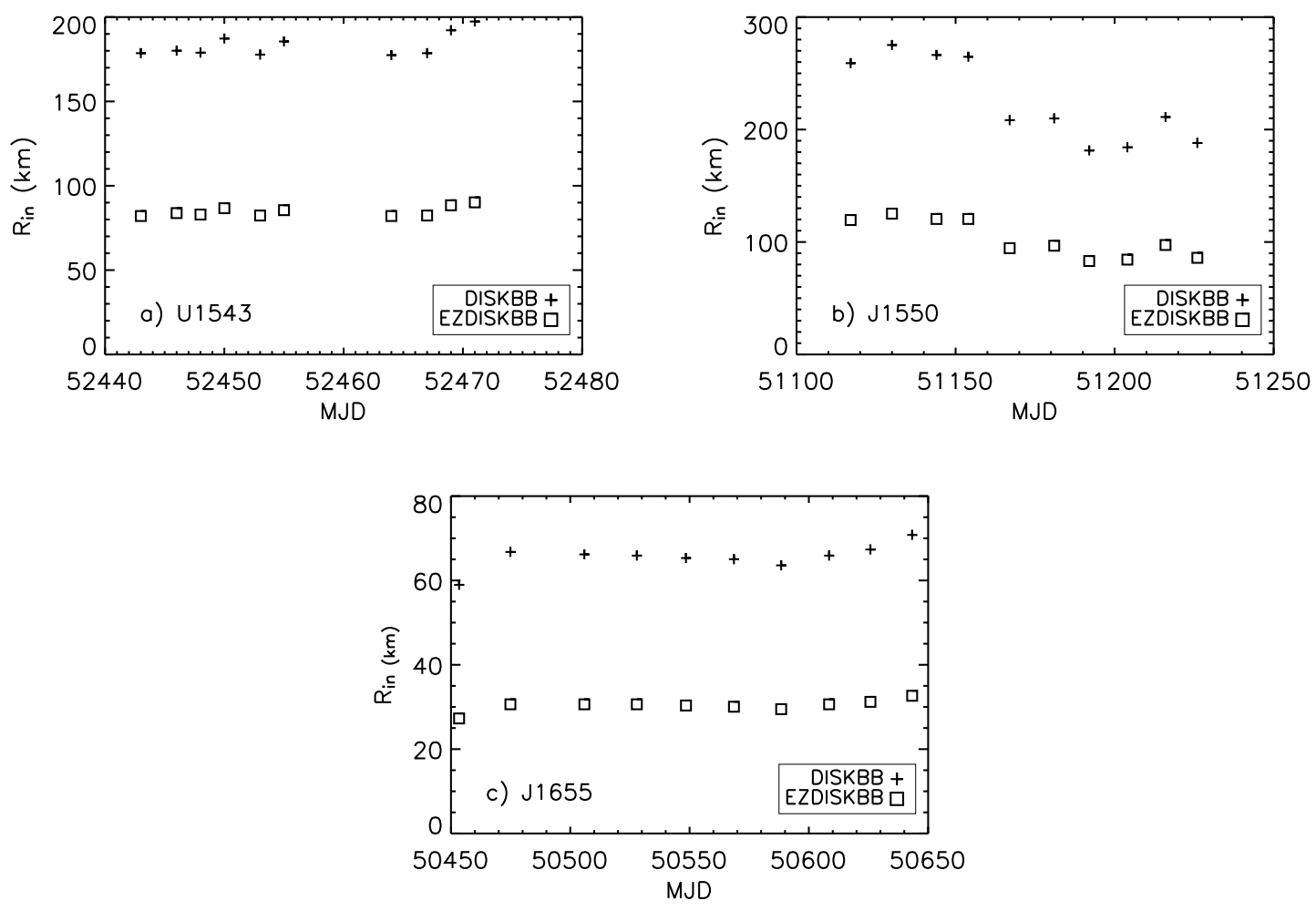

Fig. 5.- $R_{\text {in }}$ versus time for the 10 spectra from each of our BHXN, assuming a spectral hardening factor of $f=1.7$. The plus symbols indicate the values of $R_{\text {in }}$ obtained from fits using diskbb, and the squares indicate $R_{\text {in }}$ values from fits using ezdiskbb. We see that ezdiskbb gives values for the inner radius of the disk that are a factor of $\approx 2.2$ smaller than those given by diskbb; this ratio is independent of $f$. Therefore, whether one uses the zero-torque or the standard-torque boundary condition has a large effect on the determination of the inner radius of the accretion disk. 

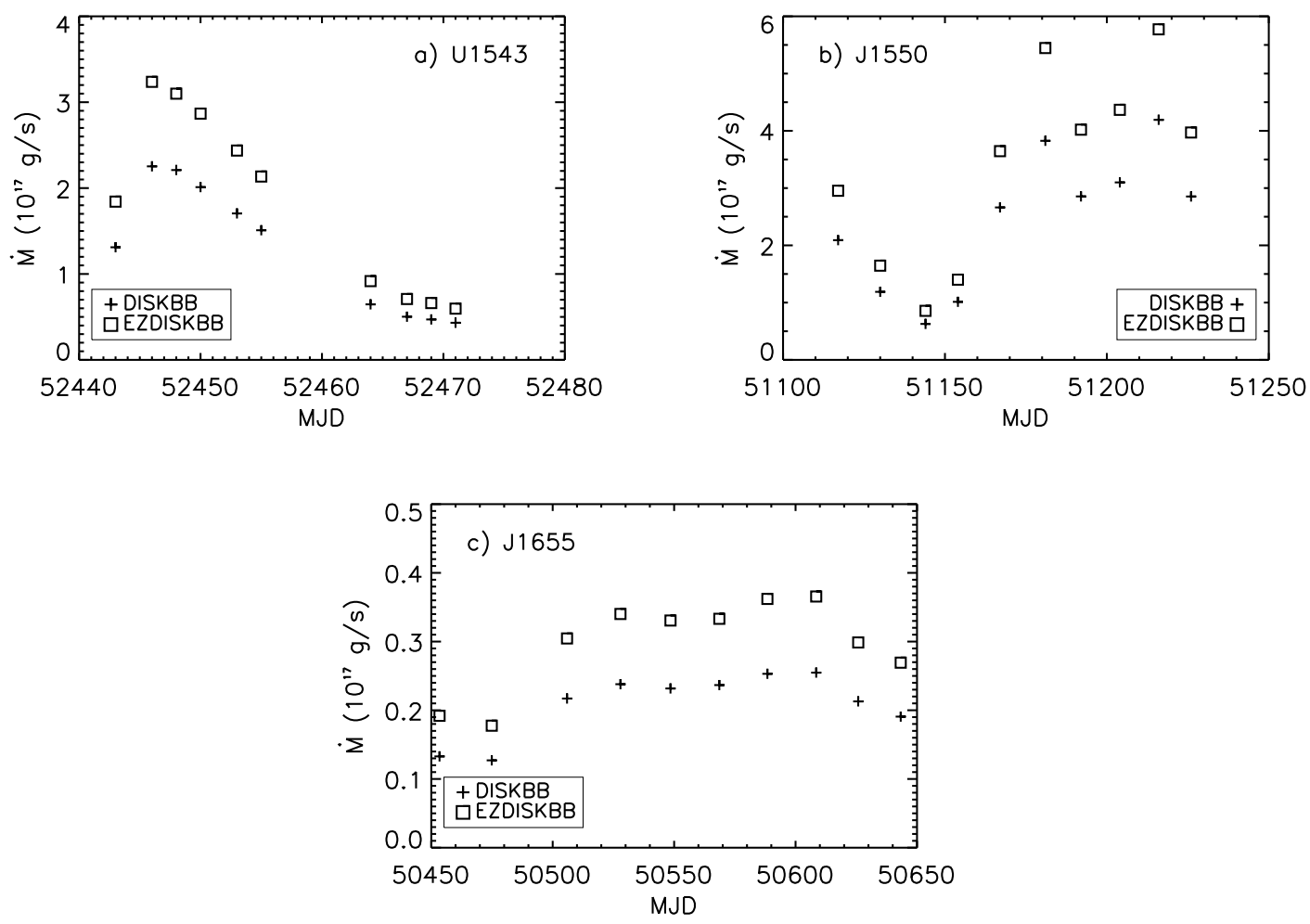

Fig. 6. $-\dot{M}$ versus time for the 10 spectra from each of our BHXN, assuming a spectral hardening factor of $f=1.7$. The plus symbols indicate the values of $\dot{M}$ obtained from fits using diskbb, and the squares indicate $\dot{M}$ values from fits using ezdiskbb. The vertical axes are in units of $10^{17} \mathrm{~g} / \mathrm{s}$. We see that ezdiskbb gives values for the accretion rate that are a factor of $\approx 1.4$ larger than those given by diskbb. The ratio is independent of $f$. 
We found that the systematic differences in the disk parameters for the two models did not significantly affect the parameters of the power-law component that we used in our fits. Likewise, we found no systematic differences between the parameters of the smedge and line components. Finally, we compared the values of $\chi^{2}$ for our fits and found that the quality of the fits was very similar for the two models. Figure 7 shows the power-law parameters and $\chi^{2}$ versus time for U1543; the other sources gave similar results. Because the power-law (and other minor) spectral parameters are scarcely affected when ezdiskbb is substituted for diskbb, and also because the computed results (Table 4) agree with the theoretical predictions, we are confident that the large differences in $R_{\text {in }}$ between the two models are genuine.

\section{Discussion}

In the preceding section, we demonstrated that the zero-torque and standard-torque boundary conditions imply systematically different values for the disk parameters. The change in $T_{\max }$ is not very large (about $5 \%$ ), but the $T_{\max }$ value obtained with diskbb is always higher than the value obtained with ezdiskbb by about this amount. The change in $\dot{M}$ is somewhat larger - a reduction of about 30\% - and this difference again appears in every spectrum. The most significant change is in the value for the inner radius of the disk: diskbb gives inner radii that are a factor of $\approx 2.2$ larger than those given by ezdiskbb.

This change in the size of $R_{\text {in }}$ has important implications, especially because the value of $R_{\text {in }}$ for an accretion disk can be used to estimate the angular momentum of the disk's central black hole. It has been shown that when a BHXN is in the high/soft state, its inner radius remains remarkably stable over time (see Fig. 5) and is therefore thought to be located near $R_{\mathrm{ms}}$ (for a review, see McClintock \& Remillard 2004). Measuring $R_{\text {in }}$ therefore allows us, in principle, to determine the approximate size of $R_{\mathrm{ms}}$. The size of $R_{\mathrm{ms}}$ depends on the dimensionless spin parameter, $a_{*} \equiv a / R_{\mathrm{g}}$, where $a=J / c M$ ( $J$ being the BH angular momentum) and $R_{\mathrm{g}} \equiv G M / c^{2}$ (e.g., Shapiro \& Teukolsky 1983). The parameter $a_{*}$ can vary between $\simeq-1$ and 1 . When $a_{*}=0$, we have a non-rotating (Schwarzschild) $\mathrm{BH}$, and $R_{\mathrm{ms}}$ $=6 R_{\mathrm{g}}$. The maximum value of $a_{*}$ is less than 1 (Thorne 1974), but when $a_{*} \simeq 1$, we have a maximally rotating $\mathrm{BH}$, and $R_{\mathrm{ms}} \simeq R_{\mathrm{g}}$. When $a_{*} \simeq-1$, the $\mathrm{BH}$ is maximally rotating in the retrograde direction, and $R_{\mathrm{ms}} \simeq 9 R_{\mathrm{g}}$. In between these extremes, $R_{\mathrm{ms}}$ decreases monotonically as a function of increasing $a_{*}$ according to the following formula (Bardeen et al. 1972):

$$
R_{\mathrm{ms}}=R_{\mathrm{g}}\left\{3+Z_{2} \mp\left[\left(3-Z_{1}\right)\left(3+Z_{1}+2 Z_{2}\right)\right]^{1 / 2}\right\}
$$



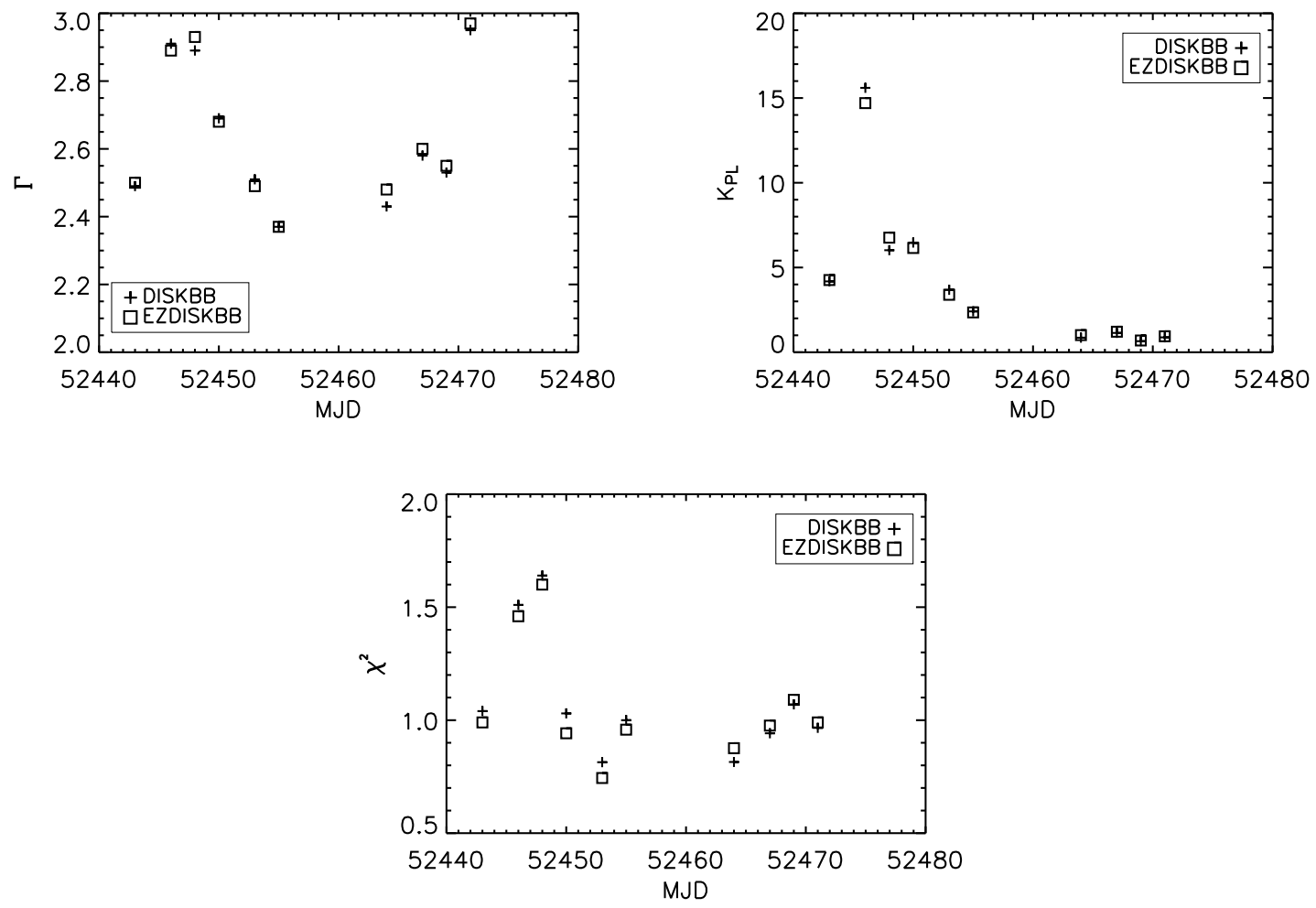

Fig. 7.- Power-law photon index $(\Gamma)$, power-law normalization $\left(K_{\mathrm{PL}}\right)$, and $\chi^{2}$ versus time for U1543. The plus symbols indicate the values given by diskbb, while the squares show the values given by ezdiskbb. As the plots show, there were no significant or systematic changes in the power-law component or the quality of our fits when ezdiskbb was substituted for diskbb.

Table 4. Diskbb Results vs. EZDiskbb Results ${ }^{\mathrm{a}}$

\begin{tabular}{ccccccccccc}
\hline \hline & \multicolumn{3}{c}{$T_{\max }$} & \multicolumn{3}{c}{$R_{\text {in }}$} & \multicolumn{4}{c}{$\dot{M}$} \\
\hline & Avg. & Max. & Min. & Avg. & Max. & Min. & Avg. & Max. & Min. \\
& Ratio & Ratio & Ratio & Ratio & Ratio & Ratio & Ratio & Ratio & Ratio \\
\hline 4U 1543-47 & 1.05 & 1.06 & 1.05 & 2.17 & 2.19 & 2.15 & 0.709 & 0.725 & 0.699 \\
XTE J1550-564 & 1.05 & 1.06 & 1.05 & 2.19 & 2.21 & 2.17 & 0.714 & 0.735 & 0.694 \\
GRO 1655-40 & 1.05 & 1.06 & 1.05 & 2.16 & 2.18 & 2.15 & 0.699 & 0.714 & 0.690 \\
Overall & 1.05 & 1.06 & 1.05 & 2.17 & 2.21 & 2.15 & 0.709 & 0.735 & 0.690 \\
\hline
\end{tabular}

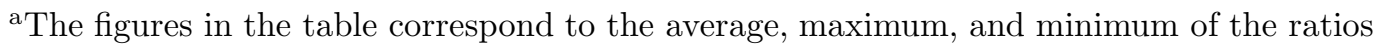
of the diskbb values to the ezdiskbb values for each parameter and object. The ratios are independent of the spectral hardening factor $f$ assumed. 


$$
\begin{array}{r}
Z_{1} \equiv 1+\left(1-a_{*}^{2}\right)^{1 / 3}\left[\left(1+a_{*}\right)^{1 / 3}+\left(1-a_{*}\right)^{1 / 3}\right], \\
Z_{2} \equiv\left(3 a_{*}^{2}+Z_{1}^{2}\right)^{1 / 2} .
\end{array}
$$

In short, $R_{\mathrm{ms}}$ decreases monotonically with increasing $a_{*}$. Since we have found that the zero-torque condition decreases our values for $R_{\text {in }}$ by a factor of 2.15 or more compared to the standard-torque condition, our estimates for $a_{*}$ will increase correspondingly by a large factor.

Figure 8 illustrates this effect by showing $R_{\mathrm{ms}}$ as a function of $a_{*}$ for each of our BHXN, using the black hole masses listed in Table 2. Also shown is the average value of $R_{\text {in }}$ for each source. We have plotted $R_{\text {in }}$ as a shaded horizontal band in order to account for the uncertainty in the value of the spectral hardening factor, which we assume to be $f=1.7 \pm 0.2$ (Shimura \& Takahara 1995). The band for each model therefore corresonds to the possible values of the effective $R_{\text {in }}$ when $f$ is between 1.5 and 1.9. The band with dashed lines corresponds to the $R_{\text {in }}$ obtained with ezdiskbb, and the band with dotted lines gives the inner radius for diskbb. Because we are assuming that $R_{\mathrm{in}}=R_{\mathrm{ms}}$, the region in which the dashed band intercepts the curve for each black hole gives the allowed range of values of $a_{*}$ for that black hole using ezdiskbb. Likewise, the intersection of the dotted band and the curve gives the allowed range of $a_{*}$ for diskbb.

We can see immediately the implications of using a model that includes the zero-torque boundary condition. For U1543 and J1550, ezdiskbb gives a range for the spin that is centered near zero, whereas diskbb gives values for $R_{\text {in }}$ that are too large to be consistent with any allowed spin of these black holes. For J1655, ezdiskbb implies a rapidly spinning black hole with $a_{*}$ between about 0.7 and 0.9 , while diskbb gives an $a_{*}$ between about -0.7 and 0.4. Sobczak (2000) used diskbb, assuming $f=1.7 \pm 0.2$, to estimate an upper limit on the spin of J1655 of about 0.7 , but we see now that if we use ezdiskbb, the spin can be greater than the Sobczak (2000) limit. This result for ezdiskbb supports past analyses that used highfrequency quasiperiodic oscillations (QPOs) to infer high spin $\left(a_{*} \approx 0.93\right)$ for J1655 (Cui, Zhang, \& Chen 1998; Strohmayer 2001). In summary, the choice of boundary condition has a large impact on the inferred spin parameter of the black holes that we have analyzed. The zero-torque boundary condition (ezdiskbb) implies substantially larger (prograde) spins than the standard-torque boundary condition (diskbb).

Since the results vary dramatically for different assumed boundary conditions, it is important to know which boundary condition is appropriate for our systems. As we discussed in $\S 2$, the zero-torque assumption is valid either (i) when we have an accretion disk around a slowly-spinning star, or (ii) when we have a thin accretion disk around a black hole (or ultracompact neutron star) with the inner edge of the disk close to the radius of the marginally 

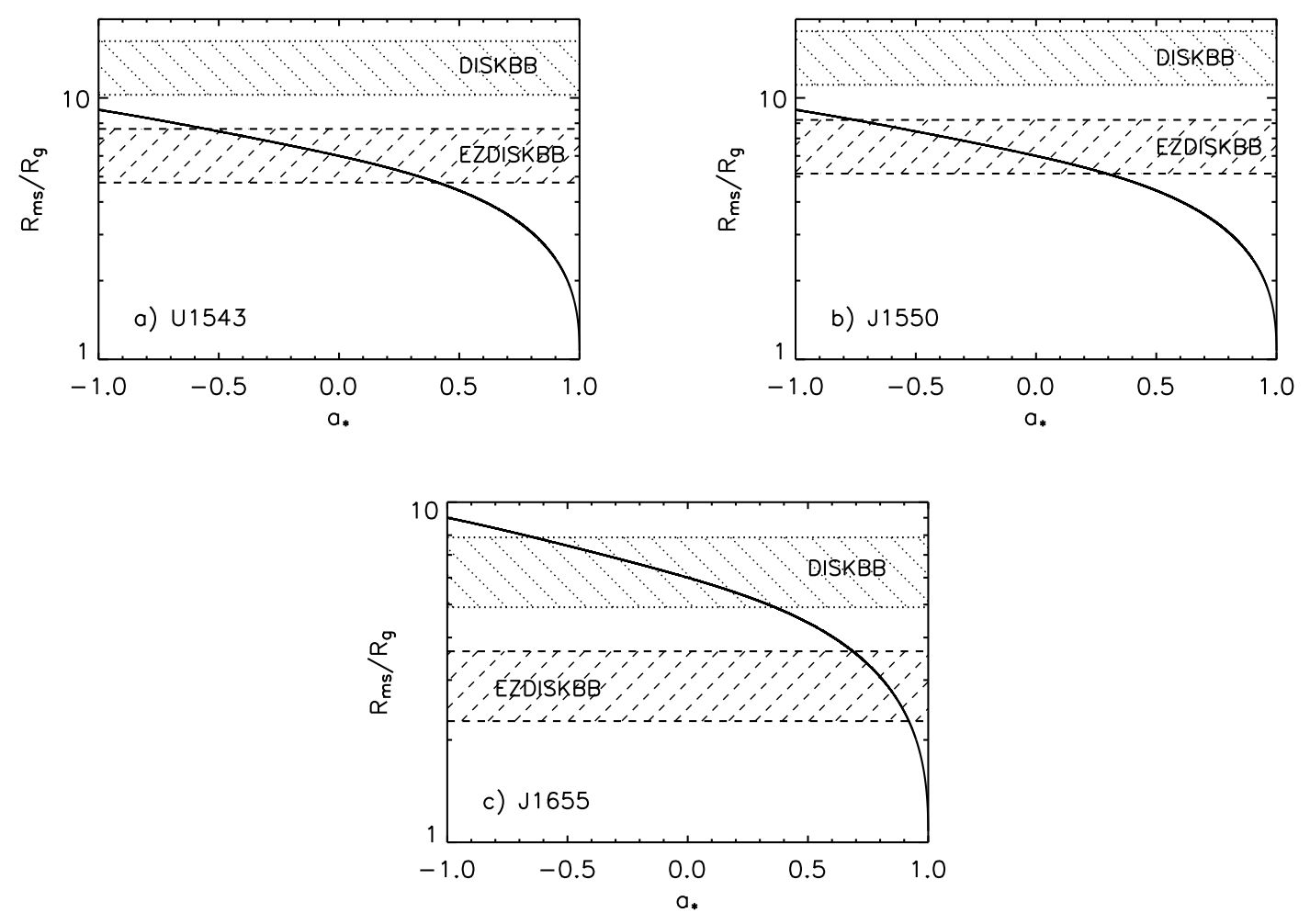

Fig. 8.- Estimates of $a_{*}$ obtained using diskbb and ezdiskbb. The solid curves show $R_{\mathrm{ms}}$ as a function of $a_{*}$. The lower and upper horizontal lines for each model correspond to the average values of $R_{\text {in }}$ when $f=1.5$ and $f=1.9$, respectively, which are the limits on the spectral hardening factor according to Shimura \& Takahara (1995). The intersection of these lines and the curve gives an estimate of the spin parameter of the black hole for that value of $f$ and the model in question. Ezdiskbb implies that U1543 and J1550 are slowly spinning, as the estimated values for $a_{*}$ are near zero. Diskbb, on the other hand, gives values for the inner radius that are too large to be consistent with any estimate for the spin parameter. For J1655, ezdiskbb indicates that $a_{*}$ should be between about 0.7 and 0.9 , while diskbb implies that $a_{*}$ is between -0.7 and 0.4 . 
stable orbit. In the black hole binaries that we have considered in this paper, the latter assumption should hold in the very high and high/soft spectral states, and perhaps also in some intermediate states.

When the accretion disk is truncated before it reaches the marginally stable orbit, as in certain models of the low and quiescent state (Narayan 1996; Esin, McClintock \& Narayan 1997; Esin et al. 1998, 2001), the gas is thought to evaporate from the optically thick geometrically thin disk to form an optically thin corona. It is possible that, in this case, the optically thick disk has a nonzero torque at its inner edge. However, there is at present no theoretical estimate of the magnitude of the torque, so it is not clear how the system is to be modeled. Another case in which there might be a significant torque on the inner edge is when a geometrically thick disk extends down to the marginally stable orbit (Afshordi \& Paczyński 2003). Indeed, MHD simulations of thick advective disks by Hawley \& de Villiers (2004) do reveal nonzero torques. However, there is no simple prescription for the magnitude of the torque, and there is no reason to believe that it would match the standard torque assumed in diskbb.

In addition, when the torque on the inner edge is nonzero, the accreting gas inside the inner edge presumably continues to experience viscous angular momentum transport and energy dissipation (Gammie 1999; Agol \& Krolik 2000). This additional source of heat will clearly contribute to the observed radiation, but its contribution will not be calculated in any thin disk model that stops the computation at the inner edge. Thus, a code like diskbb is intrinsically incomplete: it implicitly assumes additional stresses and dissipation inside the inner edge but it does not calculate the corresponding emission. In contrast, ezdiskbb is internally consistent, at least for black hole accretion. Since it assumes zero torque at the inner edge, there is no additional source of radiation inside the inner edge, and therefore the code does not make any error by neglecting the gas inside $R_{\text {in }}$.

The results shown in Figure 8 depend sensitively on the assumed value of the spectral hardening parameter $f$. In Figure 9 we show further estimates of $a_{*}$ assuming no spectral hardening, i.e., $f=1$, which is probably not a realistic assumption for any of the three sources considered herein. The values for $R_{\text {in }}$ appear here as single lines rather than bands because we are assuming a single value for $f$. In this case, our values for $R_{\text {in }}$ decrease compared to when $f=1.7$, and our estimated values for $a_{*}$ increase correspondingly. However, we still see a significant discrepancy between ezdiskbb and diskbb. Ezdiskbb now gives estimates of $a_{*}$ for U1543 and J1550 that are close to 0.9, meaning that these black holes would be very rapidly spinning. This estimate for the spin of J1550 agrees well with independently estimated values of the spin that were made using QPOs and iron lines (Miller et al. 2001). Diskbb, however, gives smaller estimates for the spins of U1543 and J1550 of about 0.5 and 
0.3, respectively. For J1655, ezdiskbb implies that $a_{*}$ is almost equal to 1, while diskbb gives a slightly smaller value of about 0.9 .

What value of $f$ is appropriate for the systems we have analysed? As we mentioned in $\S 2$, the non-blackbody effects in the emitted spectrum cannot be fully described by a single parameter $f$ - in reality, the modification to the blackbody emission is likely to be different at each photon energy. However, if we consider a simple overall measure of the spectral modification, e.g., the shift in the position of the spectral peak, then it is possible to describe the modification crudely with a single number. This is the philosophy behind the use of $f$. Shimura \& Takahara (1996) considered the effects of Comptonization and concluded that, under conditions of interest in X-ray astronomy, the change in the spectrum can be described by shifting the temperature in the blackbody formula (which determines the position of the peak) upward by $f \sim 1.5-1.9$ (average value $f \sim 1.7$ ), and at the same time changing the normalization of the blackbody formula so as to keep the flux the same.

Another relevant situation is when the escaping radiation is powered by energy released deep inside the radiating gas at an optical depth of several. This might be relevant to optically thick accretion disks if the viscous energy dissipation rate is proportional to the local density or pressure of the gas. If the gas has both absorptive and scattering opacity, then the emerging radiation will have a color temperature $T_{\text {color }}$ larger than the effective temperature $T_{\text {eff }}$ (e.g., Rybicki \& Lightman 1979). If the opacities are independent of photon energy, then the shape of the spectrum continues to be blackbody-like. However, if the opacities vary with energy (the usual case), then the spectral shape is also modified, as may be seen in the neutron star spectra calculated by Zavlin et al. (1996). For simplicity, we might wish to ignore the change in the shape of the spectrum and attempt to summarize the effects by a single parameter $f$ that describes the shift in the peak of the spectrum. As Figure 6 of McClintock et al. (2004) shows, the peak shifts by a factor of $\sim 1.65$, which is close to the value of 1.7 which we have used in most of our analysis.

\section{Summary and Conclusions}

We have confirmed that diskbb, the very widely used MTB model in the current version of XSPEC, assumes a nonzero torque at the inner boundary of the accretion disk, which is

not in accord with the classic and current literature on thin-disk accretion. We have therefore created a new model, ezdiskbb, which assumes zero torque at the inner edge of the disk. We have fitted spectra from three well known BHXN with both diskbb and ezdiskbb to compare the effect of using the different boundary conditions, and we have found that diskbb gives values for the inner radius of the disk in every spectral fit that are always a factor of $\approx 2.2$ 

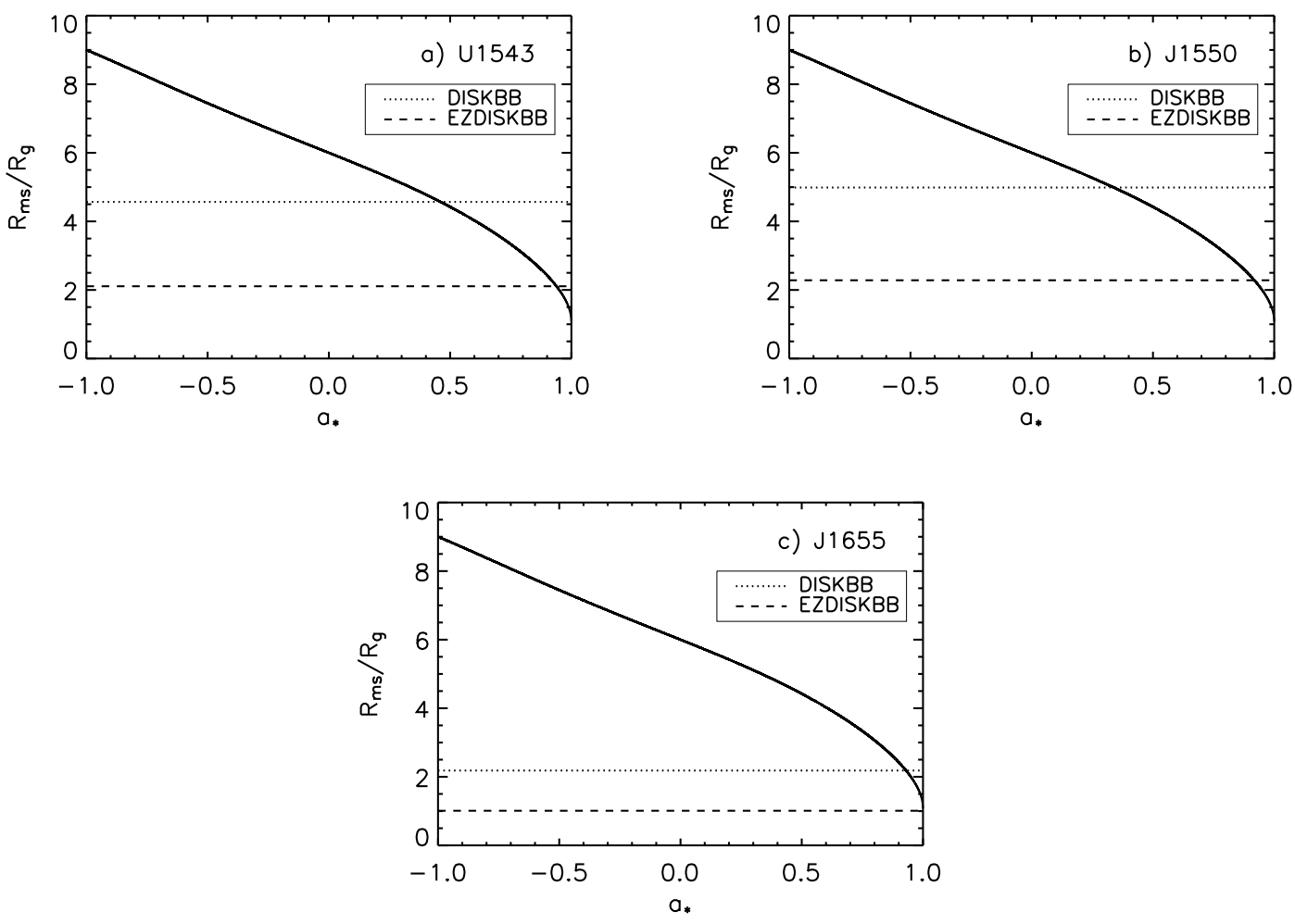

Fig. 9.- Same as Figure 8 except that a spectral hardening factor of $f=1$ is assumed, which implies that the values of $R_{\text {in }}$ are smaller and that each model corresponds to a single value of $R_{\text {in }}$. We still see a large discrepancy between the values of $a_{*}$ inferred from ezdiskbb and diskbb. Ezdiskbb implies an $a_{*}$ of about 0.9 for both U1543 and J1550, while diskbb gives estimates of about 0.5 for U1543 and 0.3 for J1550. Using QPOs and Fe K lines, Miller et al. (2001) have estimated that J1550 has high spin; ezdiskbb agrees with this result much better than diskbb. For J1655, both models indicate high spin, with ezdiskbb giving an $a_{*}$ of almost 1 and diskbb giving an $a_{*}$ of about 0.9 . 
greater than those given by ezdiskbb. We have also shown that a change in the size of the inner radius of the disk of this magnitude has significant implications when estimating the spin parameter of the disk's central black hole.

In many ways, ezdiskbb is functionally equivalent to the familiar MTB model diskbb. For example, both models are straighforward to use, are very widely applicable, and their parameter sets are identical. We have clearly outlined ezdiskbb's underlying assumptions, and we have tested it thoroughly. The theoretical arguments for fitting with ezdiskbb are based on the analysis of Afshordi \& Paczyński (2003), which indicates that the torque at the inner edge of the accretion disk is small when the disk is thin and the inner edge of the disk is close to $R_{\mathrm{ms}}$. We therefore recommend that ezdiskbb be used to analyze spectra for BHXN, especially in the high/soft state, as well as in the very high, and some of the intermediate states, in which the inner edge of the disk should be close to $R_{\mathrm{ms}}$. However, there is still some debate about the magnitude of the torque at the inner edge of the disk. For example, Hawley \& De Villiers (2004) argue that the torque in the inner disk region does not go to zero, but their simulations correspond to a relatively geometrically thick disk for which the Afshordi \& Pacsyński (2003) argument does not apply. Because the question is still open, ezdiskbb provides a useful means of comparison between the zero-torque and standard-torque assumptions. For ease and simplicity, results for the inner radius obtained with diskbb can be divided by a factor of $\approx 2.2$ in order to estimate the inner radius that would be obtained using the zero-torque inner boundary condition. Similarly, mass accretion rate estimates obtained with diskbb may be multiplied by $\approx 1.4$ to obtain the corresponding rates for the zero-torque boundary condition.

The results obtained with either diskbb or ezdiskbb are quite sensitive to the assumed value of the spectral hardening factor $f$. We have presented arguments in $\S 4$ why the choice $f=1.7$ is reasonable. However, until more accurate disk atmosphere models become available, this factor will represent a rather large uncertainty in results derived from fitting data.

The authors would like to acknowledge K. Makishima for his forthcoming and helpful discussions on diskbb. They also acknowledge Andrzej Zdiarski and Philip Kaaret for their important discussions, as well as Ron Remillard for his assistance with the BHXN lightcurves, and Keith Arnauld for his help with implementing ezdiskbb in XSPEC. This work has made use of the information and tools available at the HEASARC Web site, operated by GSFC for NASA, and was supported in part by NASA grants NAG5-9930 and NAG5-10780 and NSF grant AST 0307433. 


\section{REFERENCES}

Afshordi, N. \& Paczyński, B. 2003, ApJ, 592, 354

Agol, E. \& Krolik, J. H. 2000, ApJ, 528, 161

Armitage, P. J., Reynolds, C. S., \& Chiang, J. 2001, ApJ, 548, 868

Arnaud, K. A. 1996, in ASP Conf. Ser. 101: Astronomical Data Analysis and Systems V., 17

Bardeen, J. M., Press, W. H., \& Teukolsky, S. A. 1972, ApJ, 178, 347

Cui, W., Zhang, S. N., \& Chen, W. 1998, ApJ, 492, 53

Esin, A. A., McClintock, J. E, \& Narayan, R. 1997, ApJ, 489, 865

Esin, A. A., Narayan, R., Cui, W., Grove, J. E., \& Zhang, S.-N. 1998, ApJ, 505, 854

Esin, A. A., McClintock, J. E., Drake, J. J., Garcia, M. R., Haswell, C. A., Hynes, R. I., \& Muno, M. P. 2001, ApJ, 555, 483

Frank, J., King, A., \& Raine, D. 1992, Accretion Power in Astrophysics (Cambridge University Press)

Gammie, C. F. 1999, ApJ, 522, L57

Gierlinski, M., Zdziarski, A. A., Poutanen, J., Coppi, P. S., Ebisawa, K., \& Johnson, W. N. 1999, MNRAS, 309, 496

Gierlinski, M. \& Done, C. 2004, MNRAS, 347, 885

Hawley, J. F. \& De Villiers, J. P. 2004, to be published in Progress of Theoretical Physics Supplement, astro-ph/0402665

Hjellming, R. M. \& Rupen, M. P. 1995, Nature, 375, 464

Jahoda, K., Swank, J. H., Giles, A. B., et al. 1996, in Proc. SPIE, Vol. 2808, EUV, X-ray, and Gamma-ray Instrumentation for Astronomy VII, ed. O. H. Siegmund \& M. A. Gummin, 59

Kluzniak, W. 1987, Ph.D. thesis, Stanford University

McClintock, J. E., Narayan, R., \& Rybicki, G. B. 2004, ApJ, in press (astro-ph/0403251) 
McClintock, J. E. \& Remillard, R. A. 2004, astro-ph/0306213

Makishima, K., Maejima, Y., Mitsuda, K., et al. 1986, ApJ, 308, 635

Miller, J. M., Wijnands, R., Homan, J., et al. 2001, ApJ, 563, 928

Mitsuda, K., Inoue, H., Koyama, K., et al. 1984, PASJ, 36, 741

Narayan, R. 1996, ApJ, 462, 136

Novikov, I. D. \& Thorne, K. S. 1973, Black Holes - Les Astres Occlus, ed. C. D. Witt \& B. S. D. Witt (Gordon \& Breach), 343

Orosz, J. A. \& Bailyn, C. D. 1997, ApJ, 477, 876

Orosz, J. A., Jain, R. K., Bailyn, C. D., McClintock, J. E., \& Remillard, R. A. 1998, ApJ, 499,375

Orosz, J. A., Groot, P. J., van der Klis, M. et al. 2002, ApJ, 568, 845

Orosz, J. A. 2003, in preparation

Paczyński, B. 1991, ApJ, 370, 597

Park, S. Q., Miller, J. M., McClintock, J. E., et al. 2003, accepted for publication in ApJ, astro-ph/0308363

Popham, R. \& Narayan, R. 1991, ApJ, 370, 604

Popham, R. \& Narayan, R. 1995, ApJ, 442, 337

Pringle, J. E. \& Rees, M. J. 1972, A\&A, 21, 1

Pringle, J. E. 1981, ARA\&A, 19, 137

Reynolds, C. S. \& Armitage, P. J. 2001, ApJ, 561, L81

Rutledge, R. E., Bildsten, L., Brown, E. F., Pavlov, G. G., \& Zavlin, V. E. 1999, ApJ, 514, 945

Rybicki, G. B., \& Lightman, A. P. 1979, Radiative Processes in Astrophysics, New York: Wiley

Shakura, N. I. \& Sunyaev, R. A. 1973, A\&A, 24, 337 
Shapiro, S. L. \& Teukolsky, S. A. 1983, Black Holes, White Dwarfs, and Neutron Stars (John Wiley \& Sons)

Shimura, T. \& Takahara, R. 1995, ApJ, 445, 780

Sobczak, G. J., McClintock, J. E., Remillard, R. A., Bailyn, C. D., \& Orosz, J. A. 1999, ApJ, 520, 776

Sobczak, G. J. 2000, PhD thesis, Department of Astronomy, Harvard University

Sobczak, G. J., McClintock, J. E., Remillard, R. A., et al. 2000, ApJ, 544, 993

Strohmayer, T. E. 2001, ApJ, 552, L49

Thorne, K. S. 1974, ApJ, 191, 507

Zavlin, V. E., Pavlov, G. G., \& Shibanov, Yu. A. 1996, A\&A, 315, 141

Zhang, S. N., Cui, W., \& Chen, W. 1997, ApJ, 482, L155 